\title{
Assessing mods in ICU - A critical appraisal
}

\author{
Rupa M. Mascarenhas ${ }^{1}$, Reshmina Clara D'Souza ${ }^{2}$, Shafeel Ibrahim K ${ }^{3}$, Sheldon Mathias ${ }^{4}$, Mohammed Ashfaque ${ }^{5 *}$ \\ ${ }^{1,3}$ Assistant Professor, ${ }^{2,4,5}$ Associate Professor, ${ }^{1,2,4,5}$ Dept. of General Surgery, ${ }^{3}$ Dept. of Medicine, ${ }^{1,3}$ AJ Institute of Medical Sciences \& \\ Research Centre, Mangalore, Karnataka, ${ }^{2,4}$ Father Muller Medical College Hospital, Mangalore, Karnataka, ${ }^{5}$ Kasturba Medical College, \\ Mangalore, Manipal Academy of Higher Education, Manipal, Karnataka, India
}

*Corresponding Author: Mohammed Ashfaque

Email: ashfaque.mohammed69@gmail.com

\begin{abstract}
The morbidity of life threatening infection is reflected by the Multiple Organ Dysfunction Syndrome (MODS), so reliable methods of assessing MODS are an important pre requisite for the evaluation and treatment of patients with sepsis. The usefulness of the sofa score has previously been validated in large cohorts of critically ill patients. It was even used as a mortality predictor and compared with other scoring systems such as APACHE. However none of the studies have used it on patients with surgical sepsis. In this study the trend of sofa scoring and its derivatives is compared in patients with surgical sepsis. All the patients were appropriately assessed and managed according to standard guidelines for the respective disease.

Materials and Methods: A prospective, non interventional study was done on multiple centres in the district of Dakshina Kannada on 120 adult patients admitted in who either presented with, or were in sepsis at the time of first examination. Between June 2018 to May 2019 . The SOFA score was collected on a daily basis. The worst value of each day was taken. The score was calculated till discharge from ICU, mortality or day 5 of admission to ICU whichever was earliest.

Results: Among 120 patients who were included in the study, majority in the surgical group were Exploratory Laprotomies secondary to Hollow viscus perforation, Diabetic foot and Necrotising fasciitis. In the NON surgical group were pneumonia, acute exacerbation of COPD, urosepsis, viral hemorrhagic fevers. A prolonged ICU stay could be predicted at the time of admission on the basis of the dysfunction of those organ systems that generally require more prolonged ICU support, that is, cardiovascular, respiratory, and neurologic systems.

Conclusion: For management, mean and maximum score help determine the severity of illness and can act as a guide for the intensity of therapy required for each patient.
\end{abstract}

Keywords: MODS, ICU.

\section{Introduction}

The morbidity of life threatening infection is reflected by the Multiple Organ Dysfunction Syndrome (MODS), so reliable methods of assessing MODS are an important pre requisite for the evaluation and treatment of patients with sepsis. In general terms outcomes in clinical trials are being used to answer one of the two big questions, whether an intervention works and if it does, whether it helps. Patients either present in frank sepsis or develop it as they course through their hospital stay, due to endogenous or exogenous reasons. The cause of sepsis thus varies, however the most common organisms responsible are Aerobic Gram Negative Bacilli, though several others are on the rise with use of broad spectrum antibiotics in the ICU setups. The issues pertaining to sepsis have been explored in depth and using extensive scoring systems, reliable predictions have been made regarding the course of the disease. Also, interventions have been implemented in an attempt to break the natural course. For example, in this dissertation we explore the outcomes of surgical sepsis with the help of scoring systems and serum albumin, and their usefulness on the predictability of morbidity and mortality. Developed initially as a sepsisrelated organ failure assessment in $1994,{ }^{1}$ the SOFA score was renamed when it was found to be applicable for both septic as well as nonseptic patients. ${ }^{2}$ This system includes six major organ systems (pulmonary, hematologic, hepatic, cardiovascular, central nervous, and renal), records the most deranged value on each day, and scores the derangement from 0 (normal) to 4 (most deranged).SOFA scores can be taken daily or on a $48 \mathrm{~h}$ basis. The best correlation of scores with the outcome in terms of morbidity and mortality is seen with maximum SOFA score and mean SOFA score. ${ }^{3}$ Published in 1995, the MODS includes six organ systems, records the most representative value of the day, and scores the abnormality from 0 (normal) to 4 (abnormal). Most prognostic scoring systems rely on a single analysis of patient data to calculate mortality risk. The importance of repeated scoring, allowing serial patient assessment and enabling measurement of resource utilization, has been noted by several groups. ${ }^{4}$ Regular data collection will also assist in the evaluation of new therapies. Organ dysfunction is a dynamic process, and the simplicity of the SOFA score and the parameters used makes repeated measures an easy task. Calculating the score is straightforward, and the use of computerized systems with automatic data collection may make it even easier SOFA scoring was initially described by Vincent et al to quantitatively and objectively assess organ dysfunction in groups/individual patients. ${ }^{32}$ The usefulness of the score has previously been validated in large cohorts of critically ill patients .It was even used as a mortality predictor and compared with other scoring systems such as APACHE. However none of the studies have used it on patients with surgical sepsis. In this study the trend of sofa scoring and its derivatives is compared in patients with surgical sepsis. All the patients were appropriately assessed and managed according to standard guidelines for the respective disease. 


\section{Materials and Methods}

A prospective, non interventional study was done on multiple centres in the district of Dakshina Kannada on 120 adult patients admitted in who either presented with, or were in sepsis at the time of first examination between June 2018 to May 2019. The patients satisfying the following criteria were included in the study. Presence of 2 or more features of SIRS, Age> 15 years, the exclusion criteria was the patients who expired during ICU stay of less than 48 hours or those who were lost to follow up.

The SOFA score was collected on a daily basis. The worst value of each day was taken. The score was calculated till discharge from ICU, mortality or day 5 of admission to ICU whichever was earliest. Data included patient demographics, diagnosis, invasive and non-invasive procedures undertaken, daily laboratory investigations, vital parameters. All investigations were those that are routinely done in surgical ICU patients. It did not involve incurring of any additional costs to the patients. Morbidity data was collected with respect to the individuals diagnosis By day 3 number of patients in the study reduced to 95 , by day 5 there were 60 patients. These 60 patients were discharged room ICU and transferred out to a step down like intensive treatment unit or ward. Seven patients were discharged against medical advice. Body fluids sent for culture included - blood, sputum, urine, tracheal tap \& pus. The suspected sites of inection were categorized as follows: Intraabdominal - peritonitis, Chest- ARDS, pneumonia skin and soft tissue necrotizing fasciitis, diabetic foot. Nosocomial - infected central line, UTI which is highly significant statistically $\mathrm{p}$ value $=<0.001$. To the clinician, the ability to follow patient progress and disease development is of value and, in this study, scores were calculated every day. Survivors and nonsurvivors followed an almost parallel SOFA score course, but the non-survivors had a more severe degree of organ dysfunction from the time of ICU admission.

\section{Results}

Minimum age of the patient is 14 yrs, Maximum age of the patient is $78 \mathrm{yrs} .75 \%$ of patients are in group of $>30 \mathrm{yrs}, 60 \%$ of patients of $>50 \mathrm{yrs}$. Among 120 patients who were included in the study, majority in the surgical group were Exploratory Laprotomies secondary to Hollow viscus perforation, diabetic foot and Necrotising fasciitis. In the NON surgical group were pneumonia, acute exacerbation of COPD, urosepsis, viral hemorrhagic fevers .However there has been no association between gender, the score and the prognosis in this study. From demographic data, it was found there has been no association between age, the score and the prognosis in this study. The most common co morbidities noted were diabetes (85\%), Hypertension (37\%), Mortality is very high in patients with comorbidity i.e $78 \%$ than without comorbidity $22 \%$. Survivors and non-survivors followed an almost parallel SOFA score course, but the non-survivors had a more severe degree of organ dysfunction from the time of ICU admission. This analysis was potentially affected by the continuous turnover of patients on the ICU, with patients leaving the unit at various times, either because they died or were discharged. We therefore analyzed a subgroup of patients who remained in the ICU for at least 2 days. In this subgroup also, a greater degree of organ dysfunction was noted in the nonsurvivors. A prolonged ICU stay could be predicted at the time of admission on the basis of the dysfunction of those organ systems that generally require more prolonged ICU support, that is, cardiovascular, respiratory, and neurologic systems. Infected patients consistently showed a greater degree of organ dysfunction for all organ systems, which supports the widely acknowledged relationship between sepsis and multiple organ failure. We were not able to identify any particular pattern of organ dysfunction in the infected patients.

\section{Discussion}

Vincent et $\mathrm{al}^{1}$ showed that mean age o the patient is $55+/-19$ years ranging from $12-95$ years., and Jain et $\mathrm{al}^{5}$ showed that more than $80 \%$ of subjects were $<55$ years of age, A study done by Vincent et al. ${ }^{1}$ The use of maximum SOFA score to quantify organ dysfunction/failure in patients admitted in intensive care showing over $64 \%$ of patients were male. Takeshi et $\mathrm{al}^{6}$ sttaed that when evaluating mortality with severe sepsis, over half of the patients were male (61.4\%). Aditi et $\mathrm{al}^{5}$ stated that the mortality rate was $39 \%$ and the mean duration of stay in the ICU was 9 days. The maximum score in survivors $(3.92 \pm 2.17)$ was significantly lower than nonsurvivors $(8.9 \pm 3.45)$. Duration of the stay did not correlate with the survival. Vincent et $\mathrm{al}^{1}{ }^{1}$ the use of maximum SOFA score to quantify organ dysfunction/failure in intensive care- The mean total maximum SOFA score was $8.2+5.4$ points, median 7 points, range $0 \pm 24$ points, and was significantly higher in non- survivors than in survivors $(13.6+/-4.8$ points versus $6.7+/-4.5$ points, $\mathrm{p}<0.001)$. Ferreira et $\mathrm{al}^{7}-$ Serial evaluation of the sofa score to predict outcome in critical ill parents. Three hundred fifty patients admitted to ICU for more than 24 hours for whom sofa score was calculated. He stated that sequential assessment of organ dysfunction during the first few days of ICU admission is a good indicator of prognosis. Independent of the initial score, an increase in sofa score during the first $48 \mathrm{hrs}$ in ICU predicts a mortality rate of atleast $50 \%$. A Study done by Jia-Kui Sun et $\mathrm{al}^{8}$ they established hypoalbuminemia in patients with surgical sepsis as the independent risk factors e very $1 \mathrm{~g} / \mathrm{L}$ decrease of albumin level below the optimal cut-off $(23 \mathrm{~g} / \mathrm{L})$ was associated with a $19.4 \%$ increase in hospital mortality and a $28.7 \%$ increase in the incidence of multiple organ dysfunction syndrome.

\section{Conclusion}

Although we initially called the score "sepsis-related," the data show that the SOFA score is not specific to sepsis and can be applied equally to the nonseptic patient SOFA score is a simple, but effective prognostic indicator and evaluator for patient progress in ICU. Day 1 SOFA can triage the patients into risk categories. For further management, mean and maximum score help determine the severity of illness and can act as a guide for the intensity of therapy required for each patient. 


\section{Conflict of Interest: None.}

\section{References}

1. Vincent JL, Moreno R Takala J. SOFA (sepsis related organ failure assessment) score to describe organ dysfunction/ failure. On behalf of the working Group on Sepsis-related Problems of the European society of intensive care Medicine. Intens Care Med 1995;22:707-10.

2. Shapiro, Nathan I. Wolfe Richard B.; Smith, Eric; Elizabeth; Bates, David W. Mortality in Emergency Department sepsis (MEDS) score: A prospectively derived and validated clinical prediction rule'". Crit Care Med 2003;31(3):670-5.

3. Minne L, Abu-Hanna A, de Jonge E. Evaluation of SOFAbased models for predicting mortality in the ICU: A systematic review. Crit Care 2008;12:R161.

4. Marshall JC, Cook DJ, Christou NV. Multiple Organ Dysfunction Score: A reliable descriptor of a complex clinical outcome. Crit Care Med 1995;23:1638-52.

5. Jain A, Palta S, Saroa R, Palta A, Sama S, Gombar S. Sequential organ failure assessment scoring and prediction of patient's outcome in Intensive Care Unit of a tertiary care hospital. J Anaesthesiol, Clin Pharmacol 2016;32(3):364-8. doi:10.4103/0970-9185.168165.

6. Umegaki T, Ikai H, Imanaka Y. The impact of acute organ dysfunction on patients mortality with severe sepsis. $J$ Anaesthesiol Clin Pharmacol 2011;27:180-4.

7. Sun J-K, Sun F, Wang X, Yuan S-T, Zheng S-Y, Mu X-W. Risk factors and prognosis of hypoalbuminemia in surgical septic patients. Lee YY, ed. Peer J. 2015;3:e1267. doi:10.7717/peerj.1267.

8. Flavio Lopes Ferreira, Daliana Peres Bota, Annette Bross, Christian Mélot, Jean-Louis Vincent. Serial Evaluation of the SOFA Score to Predict Outcome in Critically Ill Patients. JAMA 2001;286(14):1754-8.

How to cite this article: Mascarenhas RM, D'Souza RC, Ibrahim SK, Mathias S, Ashfaque M. Assessing mods in ICU - A critical appraisal. Indian J Anat Surg Head Neck Brain 2019;5(2):61-3. 